\title{
Design of a Novel Ultrawide Stopband Lowpass Filter Using a DMS-DGS Technique for Radar Applications
}

\author{
Ahmed Boutejdar, ${ }^{1}$ Ahmed A. Ibrahim, ${ }^{2}$ and Edmund P. Burte ${ }^{3}$ \\ ${ }^{1}$ Microwave Engineering Department, University of Magdeburg, 39106 Magdeburg, Germany \\ ${ }^{2}$ Electronic and Communication Engineering Department, Minia University, Minia 61519, Egypt \\ ${ }^{3}$ Micro and Sensor Department, University of Magdeburg, 39106 Magdeburg, Germany
}

Correspondence should be addressed to Ahmed Boutejdar; ahmed.boutejdar@ovgu.de

Received 18 June 2015; Accepted 31 August 2015

Academic Editor: Giancarlo Bartolucci

Copyright (C) 2015 Ahmed Boutejdar et al. This is an open access article distributed under the Creative Commons Attribution License, which permits unrestricted use, distribution, and reproduction in any medium, provided the original work is properly cited.

\begin{abstract}
A novel wide stopband (WSB) low pass filter based on combination of defected ground structure (DGS), defected microstrip structure (DMS), and compensated microstrip capacitors is proposed. Their excellent defected characteristics are verified through simulation and measurements. Additionally to a sharp cutoff, the structure exhibits simple design and fabrication, very low insertion loss in the pass band of $0.3 \mathrm{~dB}$ and it achieves a wide rejection bandwidth with overall $20 \mathrm{~dB}$ attenuation from $1.5 \mathrm{GHz}$ up to $8.3 \mathrm{GHz}$. The compact low pass structure occupies an area of $(0.40 \lambda g \times 0.24 \lambda g)$ where $\lambda g=148 \mathrm{~mm}$ is the waveguide length at the cut-off frequency $1.1 \mathrm{GHz}$. Comparison between measured and simulated results confirms the validity of the proposed method. Such filter topologies are utilized in many areas of communications systems and microwave technology because of their several benefits such as small losses, wide reject band, and high compactness.
\end{abstract}

\section{Introduction}

With the rapid progress in modern communications systems, design goals such as compact size, low cost, good quality factor, and high performance components are highly considered. To achieve these targets, many filtering structures as open-circuited stubs, hi-low impedances, parallel coupled, and end coupled filters have been investigated. Nevertheless these methods keep the satisfactory results unattainable. In order to approach the desired results, a DGS-DMS filter could be an effective solution. Due to their improved performance characteristics, many filter techniques and methodologies have been proposed and successfully realized. Defected ground structures (DGSs) with and without periodic array have been realized by etching a pattern in the backside of the metallic ground plane to obtain the stopband effect [1-13]. DGS often consisted of two large defected areas and a narrow connecting slot channel, which corresponds to its equivalent L-C elements [14]. The DGS with periodic or nonperiodic topology leads to a reject band in some frequency range due to the slow wave effect, as a result of increasing the effective capacitance and inductance of the transmission line. In general DMS-unit [15-18] is used as a complementary element for the DGS-unit to achieve required filter response. DMS compared to DGS is etched on the microstrip line and exhibits same frequency behavior. Additionally, the design is simpler than the DGS and is more easily integrated with other microwave circuits. Moreover, it has an effective reduced circuit size compared to DGS.

In this paper, a new compact microstrip low pass filter using coupled DMS, DGS resonators, and compensated capacitors is reported. The compensated capacitors are added on the top layer in order to get a sharp transmission domain and to regenerate transmission zeros to obtain a large reject band. Dimensions of the microstrip capacitors were computed according to the desired equivalent circuit, and using Richard's-Kuroda transformation and TX-Line software [19]; afterwards they were optimized by AWR EM simulator [20]. The measured results agree well with simulated results. The DGS-DMS technique (see Figure 1) in this research can be applied in microwave coupler, antennas, and in MRI technology. 


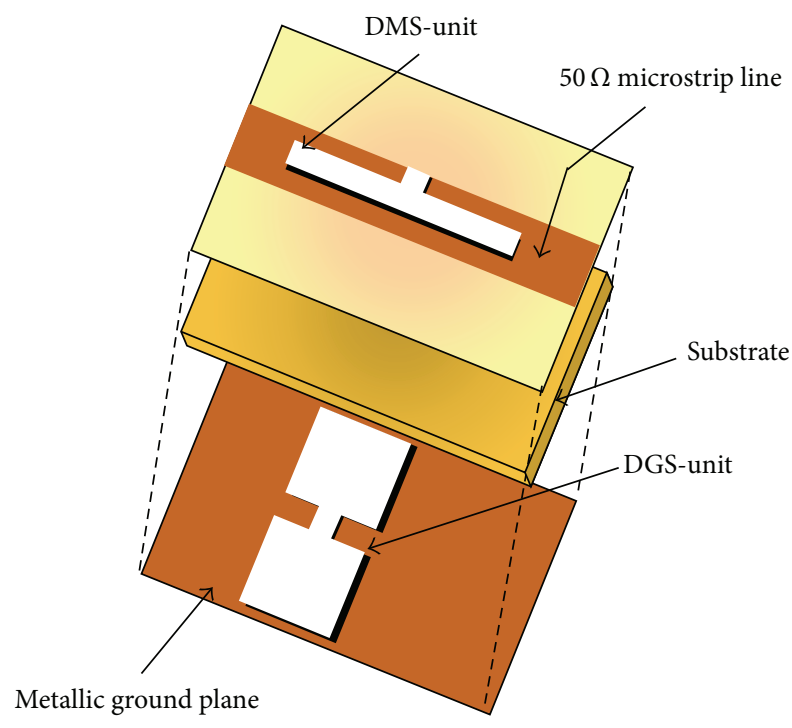

FIgURE 1: Three-dimensional view of the DMS-unit and DGS cell.

\section{Characteristics and Modeling of DMS Resonators}

Top layer of Figure 1 shows the proposed DMS cell, which is composed of wide and narrow etched sections in the feed line placed on the top layer. The extremes of this resonator are connected through microstrip line with SMA connectors. The widths of the microstrip lines at port 1 and port 2 are designed to match the characteristic impedance of $50 \Omega$. The etched surface presents the capacitance, while the arms correspond to the inductance. The DMS cell acts as a band stop element with a resonance frequency of $4.8 \mathrm{GHz}$ and an insertion loss of $-0.5 \mathrm{~dB}$ as shown in Figure 4 . The structure has been designed on RO4003 substrate with a relative dielectric constant $\varepsilon_{r}=3.38$ and thicknesses $h=0.813 \mathrm{~mm}$ and a loss tangent of 0.0027 . The equivalent circuit of the DMS cell acts as a parallel LC resonator as shown in Figure 2.

The values $R, L$, and $C$ of the circuit parameters can be computed using result that is matched to the onepole Butterworth-type low pass response [7]. Furthermore, radiation effects are more or less neglected. The reactance values of DMS and filter first order can be expressed as

$$
X_{\mathrm{LC}}=\left[\omega_{0} C\left(\frac{\omega_{0}}{\omega}-\frac{\omega}{\omega_{0}}\right)\right]^{-1} .
$$

The series inductance (reactance) of one-pole Butterworth low pass filter can be derived as follows:

$$
X_{L}=\omega L=\omega\left(\frac{g_{1} Z_{0}}{\omega_{g}}\right),
$$

where $\omega_{0}, \omega_{g}, Z_{0}$, and $g_{1}$ are the resonant frequency, cutoff frequency, the scaled characteristic impedance, and prototype value of the Butterworth-type LPF, respectively. By matching the two previous reactance values, the parallel

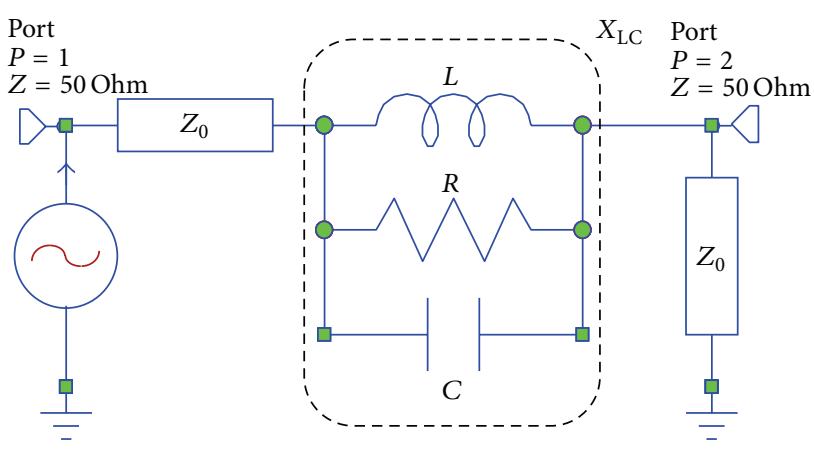

FIGURE 2: Equivalent circuit of the DMS/DGS-unit.

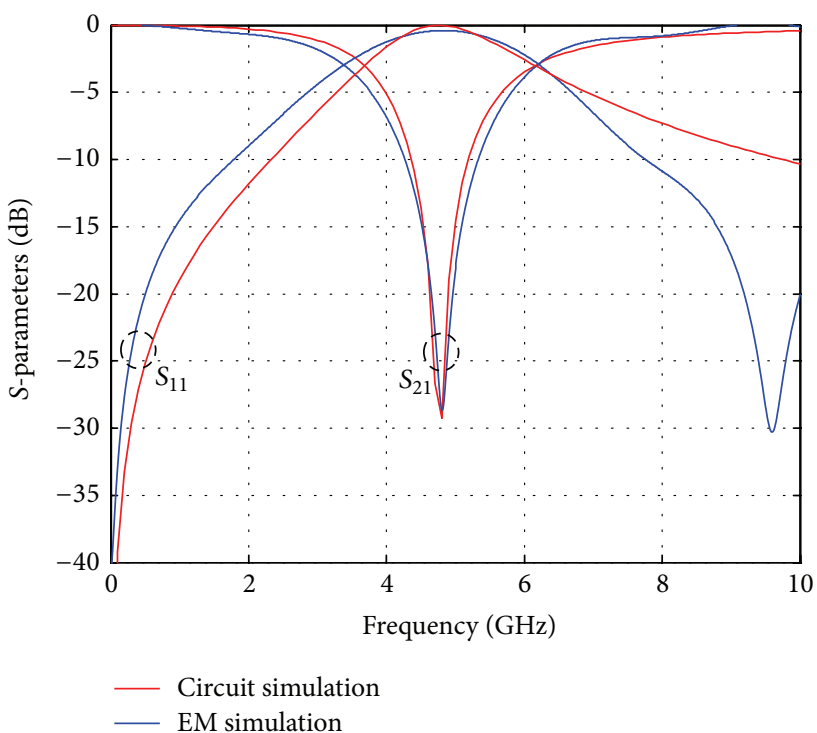

FIGURE 3: $S$-parameters of the DMS-element and its equivalent circuit.

capacitance and the inductance of the equivalent DMS-circuit can be derived using the following equations:

$$
\begin{aligned}
C & =\frac{\omega_{c}}{2 Z_{0}\left(\omega_{0}^{2}-\omega_{c}^{2}\right)}, \\
L & =\frac{1}{\omega_{0}^{2} C}, \\
R & =\frac{2 Z_{0}}{\sqrt{1 /\left|S_{11}\left(\omega_{0}\right)\right|^{2}-\left(2 Z_{0}\left(\omega_{0} C-1 / \omega_{0} L\right)\right)^{2}}-1} .
\end{aligned}
$$

The computed values of parameters $C, L$, and $R$ are, respectively, $0.64 \mathrm{pF}, 1.73 \mathrm{nH}$, and $7.42 \mathrm{k} \Omega$. The simulation results of the investigated EM structure and its corresponding circuit are illustrated in Figure 3, which shows identical values of 3 $\mathrm{dB}$ cutoff frequency $\left(f_{c}\right)$ and pole frequency $\left(f_{p}\right)$ at $3.37 \mathrm{GHz}$ and $4.83 \mathrm{GHz}$, respectively. The transmit band shows an insertion loss pass of $0.5 \mathrm{~dB}$. All dimensions of DMS-unit are depicted in Table 1. The proposed DMS resonator is shown in Figure 4. 
TABLE 1: Dimensions of the defected microstrip structure- (DMS-) element.

\begin{tabular}{lc}
\hline Dimensions of DMS-unit & Values $(\mathrm{mm})$ \\
\hline$h$ & 0.50 \\
$p$ & 1.88 \\
$g$ & 0.40 \\
$k$ & 0.60 \\
$l$ & 9.50 \\
\hline
\end{tabular}

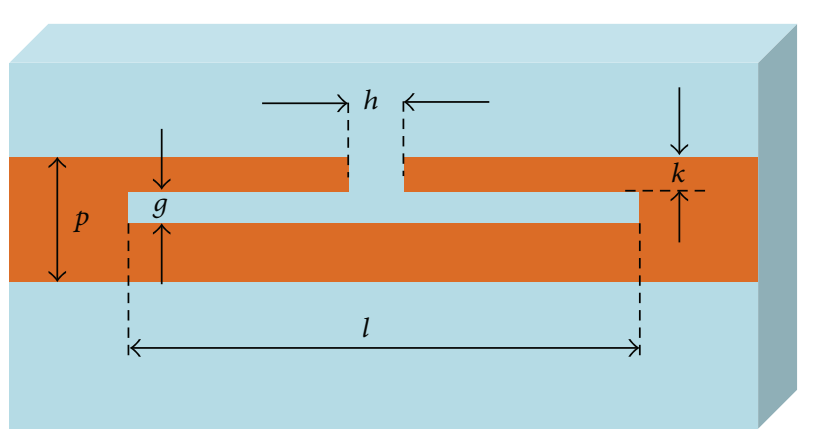

FIGURE 4: Layout of the DMS-element.

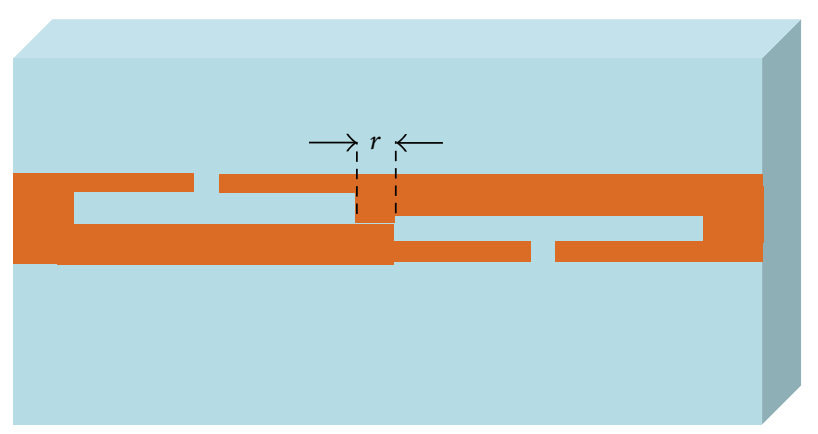

FIGURE 5: Layout of the cascaded DMS-band stop filter.

\section{Design of Band Stop Filter Using Cascaded DMS}

A new BSF was designed using two cascaded DMS resonators, which are positioned one to the other by 180 degrees and are directly connected with the ports through $50 \Omega$ microstrip lines. Figure 5 shows the $3 \mathrm{D}$ view of the proposed BSF. The geometry of each DMS-unit is equal to the dimensions indicated in Table 1, while the microstrip distance $(r)$ between two DMS resonators is $0.5 \mathrm{~mm}$. The $50 \Omega$ feed line has a line width of $w$. The band stop structure is simulated and optimized by using Microwave Studio CST [21], Microwave Office AWR. The dimensions are calculated using filter theory, TX-Line software, and EM simulator. Figure 6 shows the designed equivalent circuit of the BSF employing circuit simulator AWR. The extracted circuit parameters are computed based on the EM simulations and empirical method and defined as follows: $L=6.2 \mathrm{nH}, C=0.77 \mathrm{pF}, R=0.51 \mathrm{k} \Omega, C_{p}=0.96 \mathrm{pH}$, and $C_{0}=3.32 \mathrm{pF}$.

The simulated results depicted in Figures 7 (a) and $7(\mathrm{~b})$ prove that the proposed filter has a $3 \mathrm{~dB}$ cutoff frequency at
2.7 $\mathrm{GHz}$ and a suppression level of $20 \mathrm{~dB}$ from 4.5 to $5.5 \mathrm{GHz}$; the insertion loss in the pass band is about $0.65 \mathrm{~dB}$. Good agreement is verified between the EM simulations and the circuit simulations.

\section{Band Stop to Low Pass Using Compensated Capacitors}

In order to demonstrate the effectiveness of the compensated microstrip capacitor in transforming a structure with band stop to low pass behaviors, the added parallel microstrip capacitors to the previous structure (Figure 5) are designed and optimized as shown in Figure 8. A new DMS low pass filter is composed of three compensated parallel microstrip capacitors, which are separated through two identical DMS resonators. All components are cascaded on the top layer and directly connected with the SMAs through the two $50 \Omega$ feed lines of width $1.88 \mathrm{~mm}$ as shown in Figure 8. The filter has been designed and simulated in order to improve the reject band and to minimize the pass band losses. The DMS low pass structure is designed for cutoff frequency at $1.55 \mathrm{GHz}$ and is simulated on the Rogers RO4003 substrate with the dielectric constant of 3.38 and thickness of $0.813 \mathrm{~mm}$. The total size of the filter is $59 \times 35 \mathrm{~mm}^{2}$. Simulations have been performed using the full-wave EM Microwave Studio CST and Microwave Office AWR.

Figures 9 and 10 represent the equivalent circuit and the Sparameters of the DMS low pass filter. According to the simulation response, we can conclude that the equivalent circuit has the characteristics of quasielliptic function, because the frequency response of the elliptic function filters is known with its generated transmission zeros in pass band and thus its high sharpness in transition response as shown in Figure 10.

The values of $R, L$, and $C$ are obtained as $4 \mathrm{k} \Omega, 1.3 \mathrm{nH}$, and $0.6 \mathrm{pF}$, respectively, after using an optimization technique, while the values of three parallel open-circuit capacitors are calculated using TX-Line software or exactly calculated using the following equation:

$$
C=\frac{1}{Z_{0 C} \omega_{c}} \sin \left(\beta_{C} l_{C}\right)+\frac{2}{Z_{0 L} \omega_{c}} \tan \left(\beta_{L} l_{L}\right),
$$

where $Z_{0 C}, \beta_{C}, l_{C}, \beta_{L}$, and $l_{L}$ are the characteristic impedance, the phase constant and the physical length of the compensated capacitor (low-impedance line), and the phase constant and the physical width of the series reactance (highimpedance line), respectively. Both series reactance values of the low-impedance line are negligible; thus the length of stub capacitors is approximated as the following:

$$
\begin{aligned}
l_{C} & =\frac{\lambda g_{C}}{2 \pi} \sin ^{-1}\left(\omega_{c} Z_{0 L} C\right), \\
W_{C} & \approx f\left(Z_{0 C}, \theta, t, h, \varepsilon_{r}\right),
\end{aligned}
$$

where $W_{C}, \lambda g_{C}, \theta, t, h$, and $\varepsilon_{r}$ are the width of the opencircuit stub capacitance, the guided wavelengths, the electrical length, the thickness of metal, thickness of the substrate, and the relative dielectric constant, respectively. As shown in Figure 10, two reflection zeros at $1 \mathrm{GHz}$ and $1.5 \mathrm{GHz}$ are 


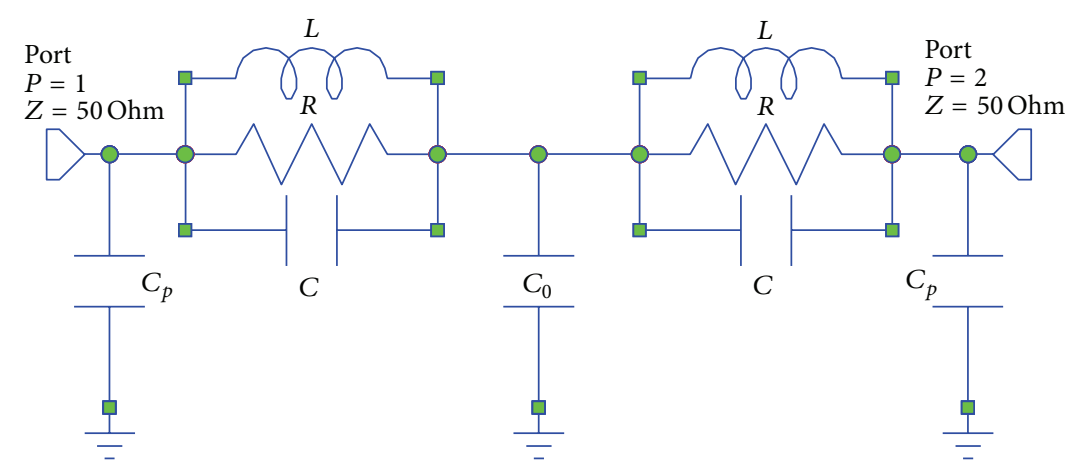

FIGURE 6: Equivalent circuit of the DMS-band stop filter.

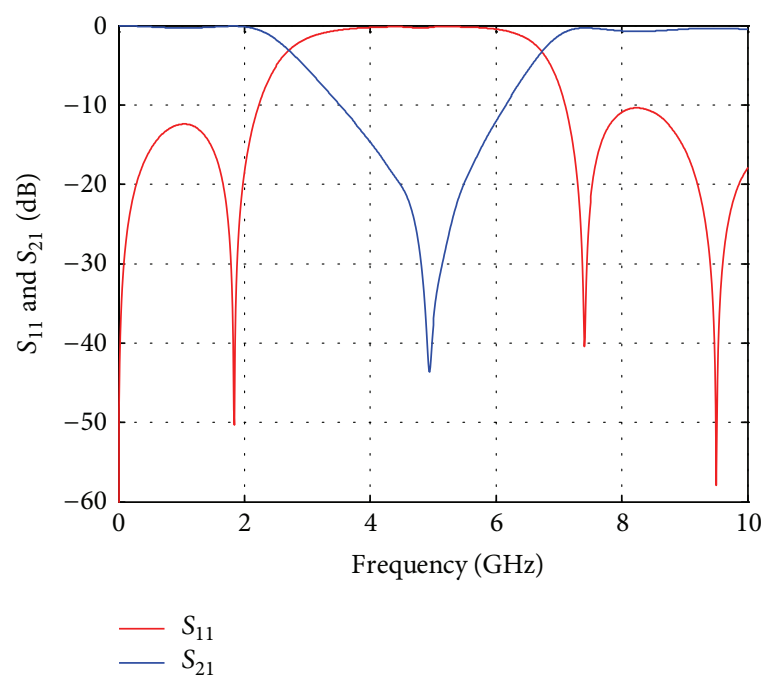

(a)

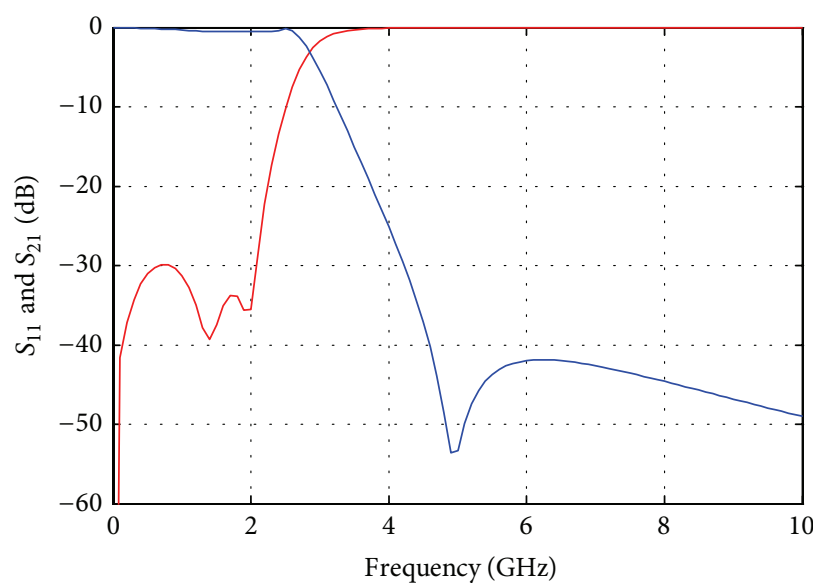

$-S_{11}$ circuit simulation $S_{21}$ circuit simulation

FIgURE 7: Simulation results of the DMS-band stop filter. (a) EM simulation, (b) circuit simulation.

TABLE 2: Dimensions of the DMS low pass filter structure.

\begin{tabular}{lc}
\hline Dimensions of DMS-unit & Values $(\mathrm{mm})$ \\
\hline$a$ & 9.05 \\
$b$ & 10 \\
$c$ & 3 \\
$e$ & 2 \\
$i$ & 20 \\
\hline
\end{tabular}

generated; thus the insertion loss is less than $0.2 \mathrm{~dB}$ from DC up to $1.55 \mathrm{GHz}$. The return loss in the pass band is less than $-12 \mathrm{~dB}$. The stopband rejection is higher than $-20 \mathrm{~dB}$ from $2.15 \mathrm{GHz}$ up to $4.25 \mathrm{GHz}$. As illustrated in Figure 10, an undesired peak appeared around the frequency of $4.4 \mathrm{GHz}$. In order to suppress this undesired harmonic, another technique based on DGS will be used. The dimensions of the proposed structure are depicted in Table 2.

\section{Improvement of the Low Pass Filter}

In order to suppress the undesired peak at $4.4 \mathrm{GHz}$ of the DMS low pass filter, a pair of DGS-units has been used. The idea is to choose DGS resonators having resonance frequency

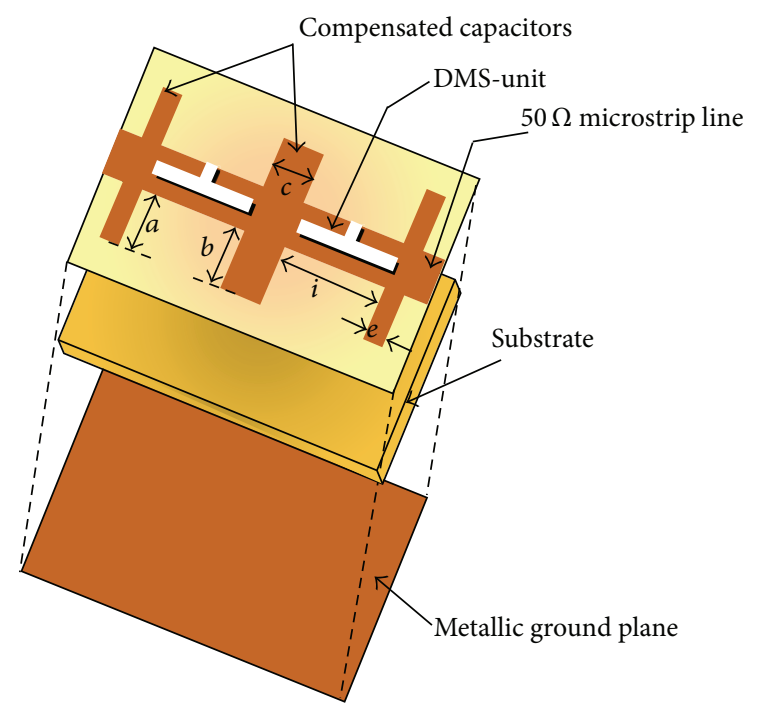

FIgURE 8: 3D view of the proposed DMS low pass filter.

around the unwanted frequency $4.4 \mathrm{GHz}$, thus to realize structure with a wide reject band. As shown in Figure 11, a multilayer structure is used to improve the performance of 


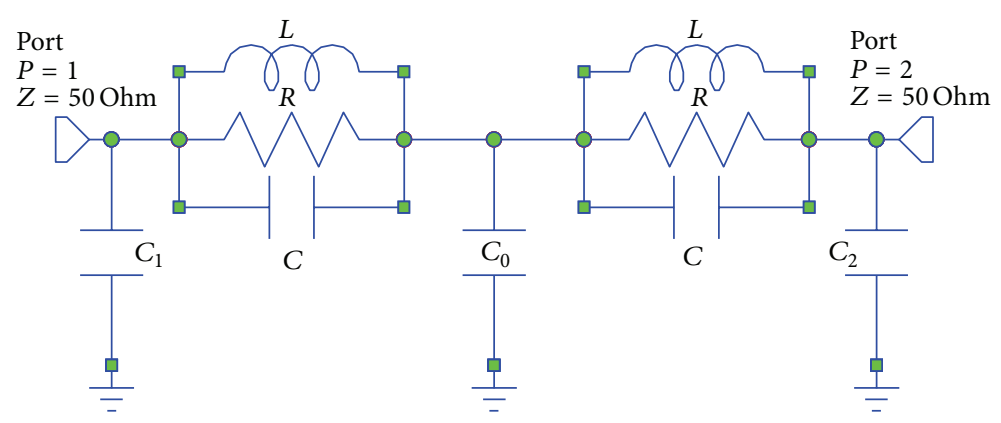

FIGURE 9: Equivalent circuit of DMS low pass filter.

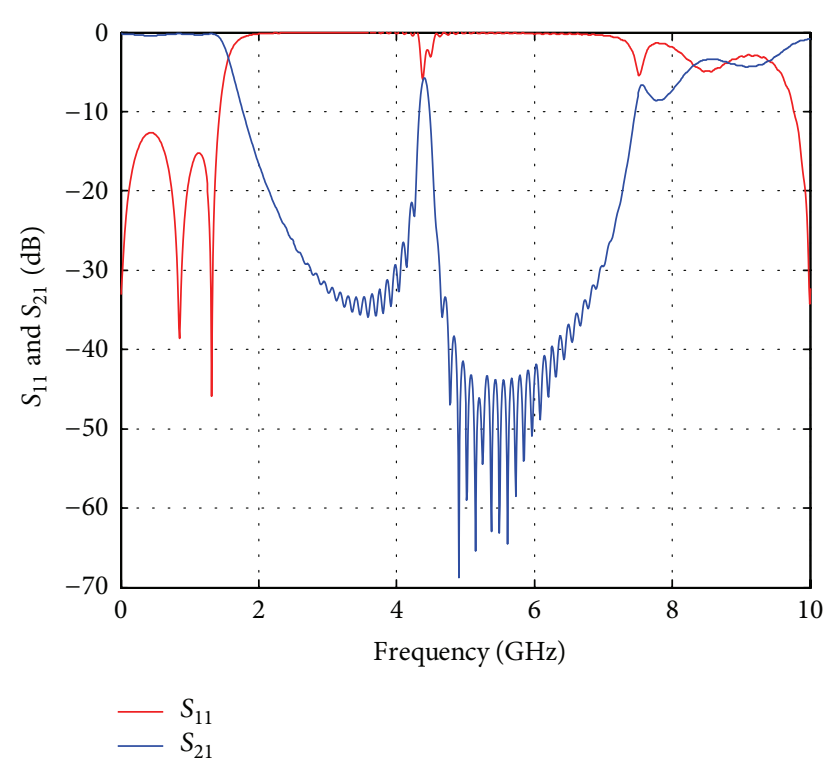

FIGURE 10: Comparison of simulated results of DMS low pass filter.

the previous low pass filter. The proposed element is similar to the DMS-unit with the difference that the new structure consists of additional two DGS-units, which are located between the microstrip capacitors as shown in Figure 11.

The dimensions of the two DGS shapes, which are etched in the ground, have been defined as follows: $s=0.6 \mathrm{~mm}$, $q=6 \mathrm{~mm}, t=10 \mathrm{~mm}$, and $z=10 \mathrm{~mm}$. The coupled distance $(d=26 \mathrm{~mm})$ between the cascaded DGS resonators is obtained based on the empirical method. This proposed geometrical idea is based on using several stacked layers and it was able to improve the performance of the filter. The proposed DGS-DMS low pass filter has been simulated on a Rogers RO4003 substrate with a relative dielectric constant $\varepsilon_{r}$ of 3.38 and a thickness $h$ of $0.813 \mathrm{~mm}$. As depicted in Figure 12, the proposed LPF behaves well in the pass band and the stopband. The filter has a $-3 \mathrm{~dB}$ cutoff frequency at $1.1 \mathrm{GHz}$, an insertion loss of $0.1 \mathrm{~dB}$, and a return loss less than $-20 \mathrm{~dB}$ in the whole pass band.

In addition, an ultrawide suppression level approximately equal to $-20 \mathrm{~dB}$ in the frequency stopband ranging from $1.5 \mathrm{GHz}$ to more than $8.5 \mathrm{GHz}$ is achieved. Simulations were performed using Microwave Office AWR and CST Microwave Studio simulators.

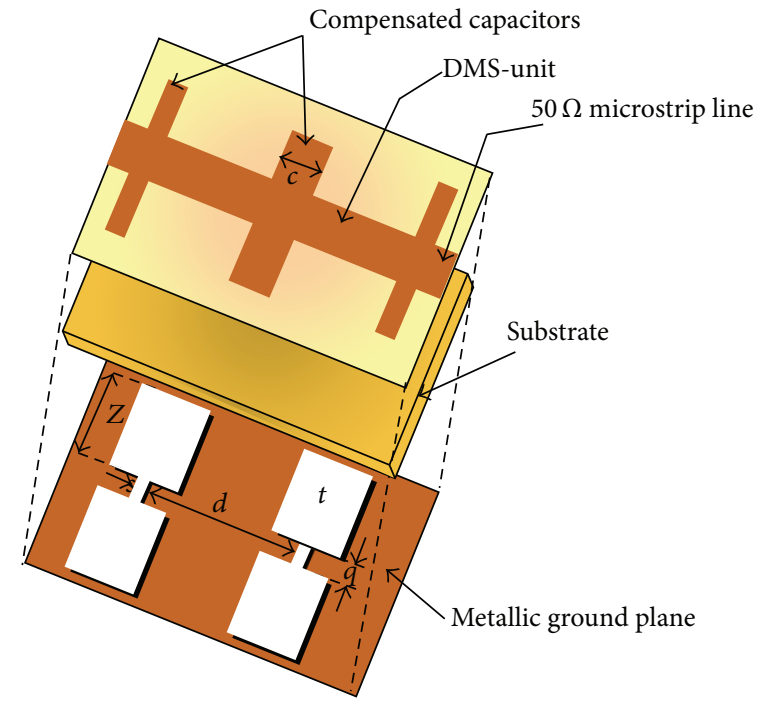

FIGURE 11: 3D view of the proposed DMS-DGS low pass filter.

\section{Field Distribution along the Filter Structure}

The investigation of this EM field distribution has an objective of showing the frequency behaviour of this proposed filter and to prove the validity of the intuitive equivalent circuit. Figure 13(a) shows the field distribution in the pass band region at the frequency of $0.5 \mathrm{GHz}$. The magnetic field is concentrated along the DMS resonators and on the $50 \Omega$ lines, while a negligible electric field appears between both poles of this DMS structure. The transmission power between both ports is magnetic. The arm of the DMS represents an inductor. Figure 13(b) shows a filter with stopband behaviour at a resonant frequency of $4 \mathrm{GHz}$. The electric and magnetic fields show same distribution densities. The electric field is concentrated between extremities of the first slot, which represents the capacity. Based on this EM field investigation, the parallel LC circuit can be an approach model for the DMS-unit.

\section{Fabrication and Measurement}

Figure 14 shows photographs of the fabricated LPF filter. The simulations of the proposed DMS-DGS-UWRB-LPF are carried out using CST Microwave Studio and AWR Microwave 


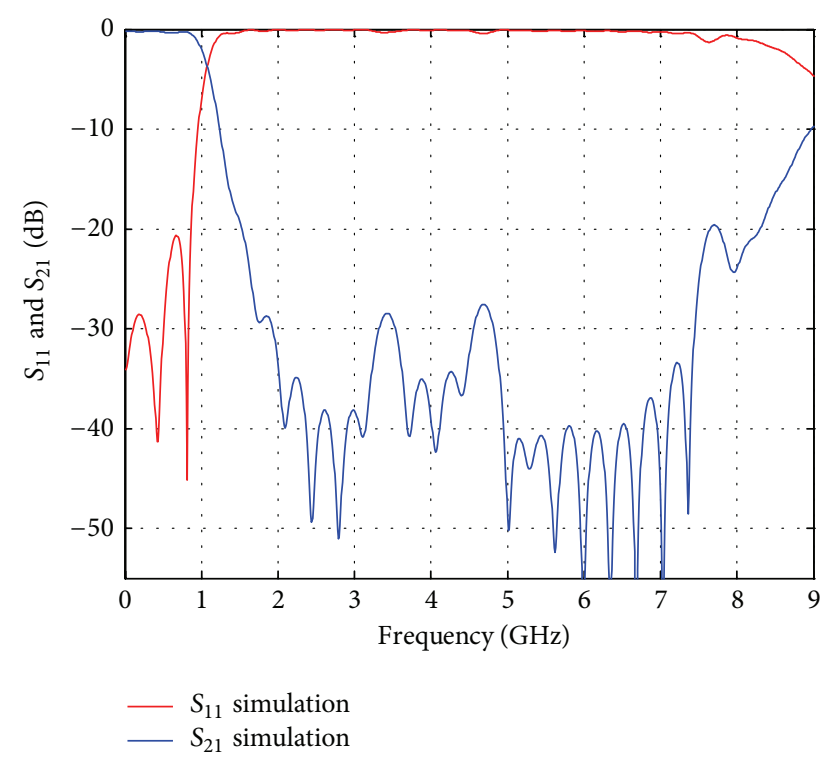

FIGURE 12: Simulation results of the proposed DMS-DGS low pass filter.

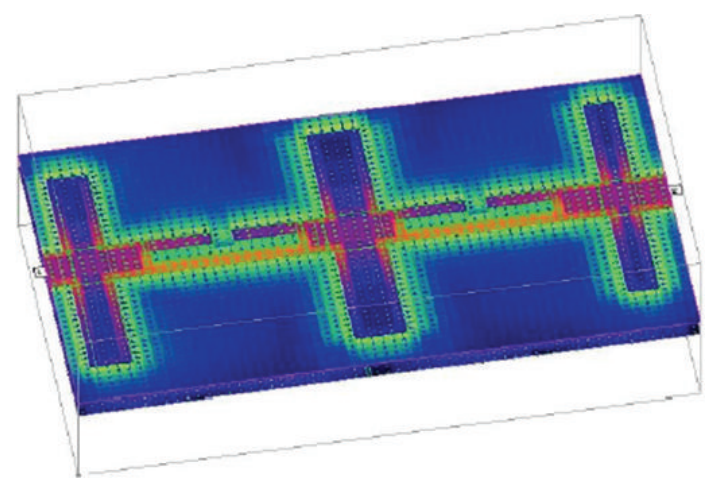

(a)

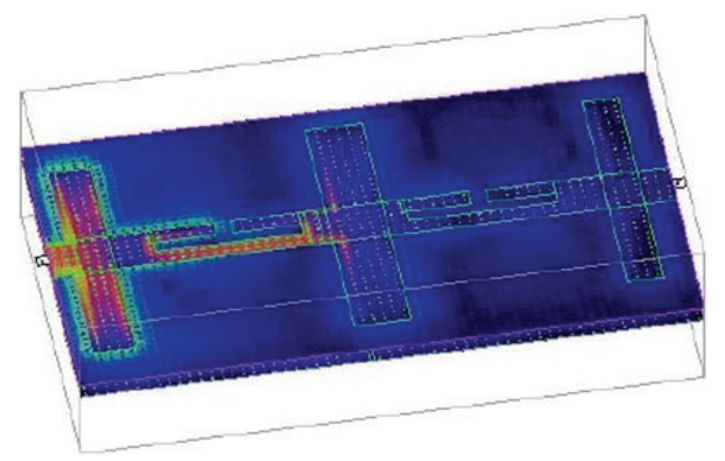

(b)

FIGURE 13: Field distribution: (a) magnetic field at $f=0.5 \mathrm{GHz}$, (b) magnetic field at $f=4 \mathrm{GHz}$.

Office. The simulation results show that the designed filter has a high sharpness factor, small losses in the pass band, and a wide reject band as shown in Figure 15. In order to verify the validity of the proposed DMS-DGS combination idea, the filter has been fabricated and measured using an HP8722D network analyzer. The LPF has been fabricated on a substrate with a relative dielectric constant $\varepsilon_{r}$ of 3.38 and a thickness

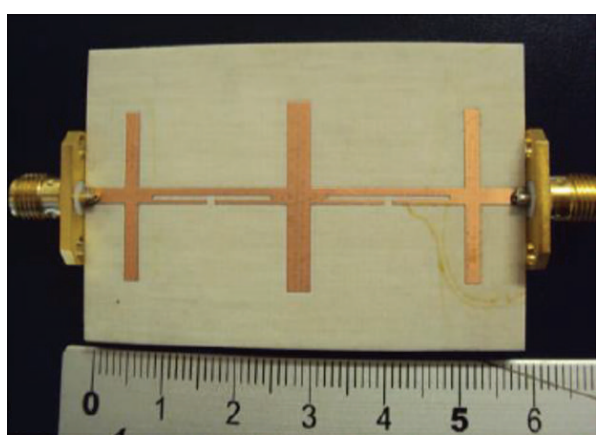

(a)

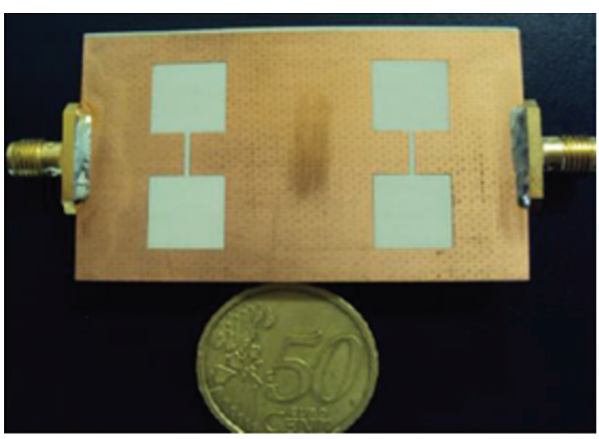

(b)

Figure 14: Photograph of the fabricated DMS-DGS LPF, (a) top layer, (b) bottom layer.

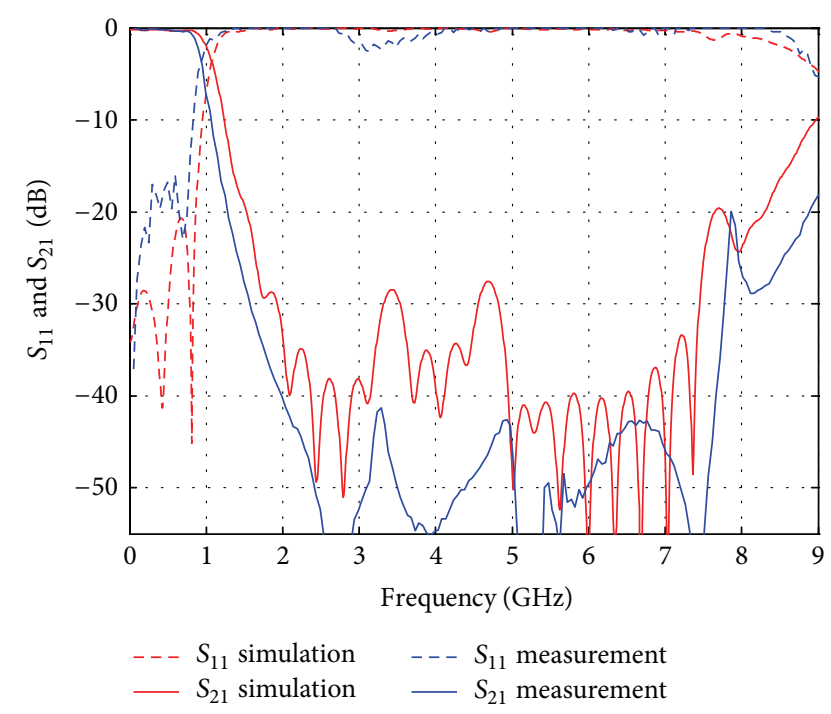

FIGURE 15: Comparison of simulation and measurement results of proposed DMS-DGS LPF.

$h$ of $0.813 \mathrm{~mm}$. The comparison between measured and simulated results is depicted in Figure 15. In the pass band, the measured insertion and return losses are less than $-0.3 \mathrm{~dB}$ and $-17 \mathrm{~dB}$, respectively. The stopband rejection is higher than $-20 \mathrm{~dB}$ from $1.3 \mathrm{GHz}$ up to $8.9 \mathrm{GHz}$ The compact low pass structure occupies an area of $(0.40 \lambda \mathrm{g} \times 0.24 \lambda \mathrm{g})$, where $\lambda g=148 \mathrm{~mm}$ is the guided wavelength at cutoff frequency. 
A very good agreement between simulations and measurements has been obtained. Some discrepancy between them can be interpreted as unexpected fabrication tolerances. The observed deviation between simulation and experimental results, which also means the loss of transmission line, has been caused by mismatching effects and the manufacturing tolerance errors.

\section{Conclusion}

In this work, a novel DMS-DGS wide stopband low pass filter has been introduced and investigated. The filter structure with strong suppression of undesired harmonic responses has been presented, which is based on stopband behaviors of the DMS and DGS cells. It is demonstrated that low insertion loss $(0.3 \mathrm{~dB})$, deep return loss (greater than $17 \mathrm{~dB})$, and a wide rejection bandwidth with overall $20 \mathrm{~dB}$ attenuation from $1.3 \mathrm{GHz}$ up to $8.9 \mathrm{GHz}$ and bandwidth with overall $40 \mathrm{~dB}$ attenuation from $1.9 \mathrm{GHz}$ up to $7.7 \mathrm{GHz}$ have been achieved in this type of filter. It has been shown that the simulated results achieved by full-wave EM were in excellent agreement with the measured ones. The newly proposed DMS-DGS-LPF and the related design method are compatible with monolithic microwave integrated circuit (MMIC) or multilayer technology and can be used in a wide range of microwave and millimeter wave applications.

\section{Conflict of Interests}

The authors declare that there is no conflict of interests regarding the publication of this paper.

\section{Acknowledgments}

The authors thank the German Research Foundation (DFG) for financial support. The authors thank Ms. Eng. Sonja Boutejdar for her assistance and help and Mr. Harald Dempewolf, the Lab Manager of the Institute for Electronics, Signal Processing and Communication (IIKT) at the University of Magdeburg, Germany, for his support.

\section{References}

[1] H. Liu, X. Sun, and Z. Li, "Novel two-dimensional (2-D) defected ground array for planar circuits," Active and Passive Electronic Components, vol. 27, no. 3, pp. 161-167, 2004.

[2] A. K. Arya, M. V. Kartikeyan, and A. Patnaik, "Defected ground structure in the perspective of microstrip antennas: a review," Frequenz, vol. 64, no. 5-6, pp. 79-84, 2010.

[3] A. Boutejdar, "Design of broad-stop band low pass filter using a novel quasi-Yagi-DGS-resonators and metal box-technique," Microwave and Optical Technology Letters, vol. 56, no. 3, pp. 523-528, 2014.

[4] F. Karshenas, A. R. Mallahzadeh, and J. Rashed-Mohassel, "Size reduction and harmonic suppression of parallel coupledline bandpass filters using defected ground structure," Applied Computational Electromagnetics Society Journal, vol. 25, no. 2, pp. 149-155, 2010.
[5] A. Boutejdar, A. Omar, and E. Burte, "High-performance wide stop band low-pass filter using a vertically coupled DGS-DMSresonators and interdigital capacitor," Microwave and Optical Technology Letters, vol. 56, no. 1, pp. 87-91, 2014.

[6] M. K. Mandal and S. Sanyal, "A novel defected ground structure for planar circuits," IEEE Microwave and Wireless Components Letters, vol. 16, no. 2, pp. 93-95, 2006.

[7] A. Boutejdar, "New method to transform band-pass to lowpass filter using multilayer- and U-slotted ground structuretechnique," Microwave and Optical Technology Letters, vol. 53, no. 10, pp. 2427-2433, 2011.

[8] H. Liu, B. Ren, X. Xiao, Z. Zhang, S. Li, and S. Peng, "Harmonic-rejection compact bandpass filter using defected ground structure for GPS application," Active and Passive Electronic Components, vol. 2014, Article ID 436964, 4 pages, 2014.

[9] M. Challal, A. Boutejdar, M. Dehmas, A. Azrar, and A. Omar, "Compact microstrip low-pass filter design with ultra-wide reject band using a novel quarter-circle dgs shape," Applied Computational Electromagnetics Society Journal, vol. 27, no. 10, pp. 808-815, 2012.

[10] A. Boutejdar, A. Batmanov, M. H. Awida, E. P. Burte, and A. Omar, "Design of a new bandpass filter with sharp transition band using multilayer-technique and U-defected ground structure," IET Microwaves, Antennas and Propagation, vol. 4, no. 9, pp. 1415-1420, 2010.

[11] G. E. Al-Omair, S. F. Mahmoud, and A. S. Al-Zayed, "Lowpass and bandpass filter designs based on DGS with complementary split ring resonators," Applied Computational Electromagnetics Society Journal, vol. 26, no. 11, pp. 907-914, 2011.

[12] Z. Pan and J. Wang, "Design of the UWB bandpass filter by coupled microstrip lines with U-shaped defected ground structure," in Proceedings of the International Conference on Microwave and Millimeter Wave Technology (ICMMT '08), vol. 1, pp. 329-332, April 2008.

[13] A. Boutejdar, A. B. A. Omar, and E. Burte, "LPF builds on quasiyagi DGS," Microwaves \& RF, vol. 52, no. 9, pp. 72-77, 2013.

[14] M. Al Sharkawy, A. Boutejdar, F. Alhefnawi, and O. Luxor, "Improvement of compactness of lowpass/bandpass filter using a new electromagnetic coupled crescent defected ground structure resonators," Applied Computational Electromagnetics Society Journal, vol. 25, no. 7, pp. 570-577, 2010.

[15] H.-H. Xie, Y.-C. Jiao, B. Wang, and F.-S. Zhang, "DMS structures stop band pass filter harmonics," Microwaves and RF, vol. 50, pp. 72-76, 2011.

[16] J.-K. Xiao and W.-J. Zhu, "New defected microstrip structure bandstop filter," in Proceedings of the Electromagnetics Research Symposium, pp. 1471-1474, Suzhou, China, September 2011.

[17] J.-K. Xiao and W.-J. Zhu, "Non-uniform defected microstrip structure lowpass filter," Microwave Journal, vol. 14, 2012.

[18] J.-K. Xiao, X. Xian, and W.-J. Zhu, "New bandstop filter using simple defected microstrip structure," Microwave Journal, vol. 11, 2011.

[19] AWR Corporation, TX-LINE: Transmission Line Calculator, AWR Corporation, San Jose, Claif, USA, 2015.

[20] Microwave Office AWR, http://web.awrcorp.com/.

[21] Microwave Studio Software, version 12, CST Corporation. 

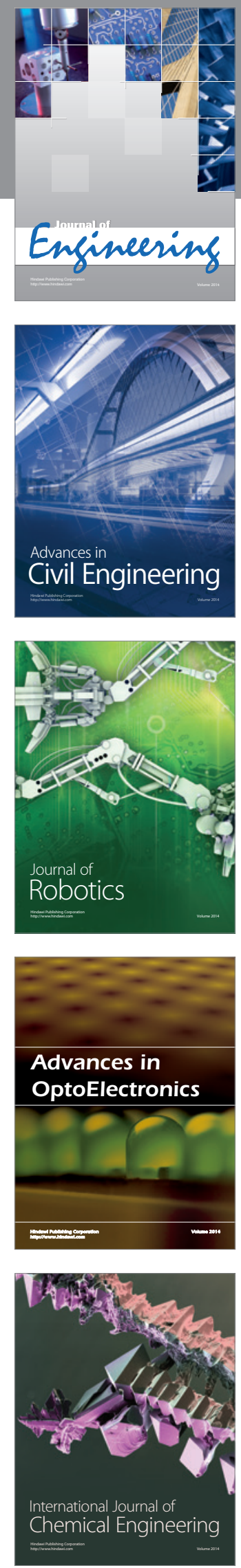

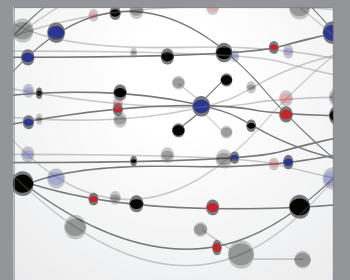

The Scientific World Journal
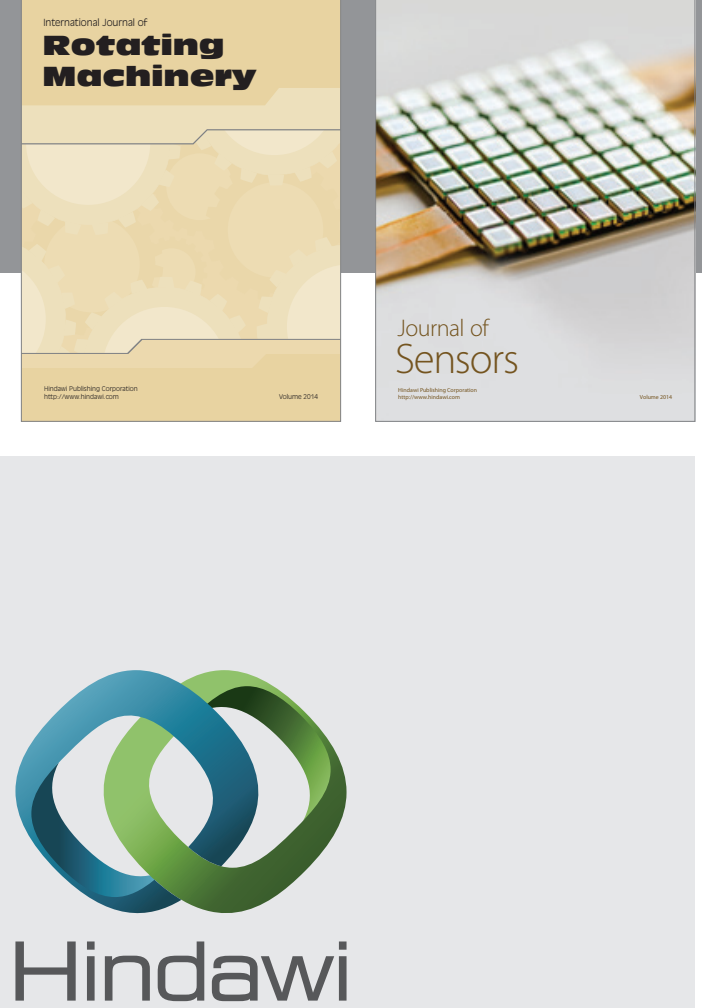

Submit your manuscripts at http://www.hindawi.com
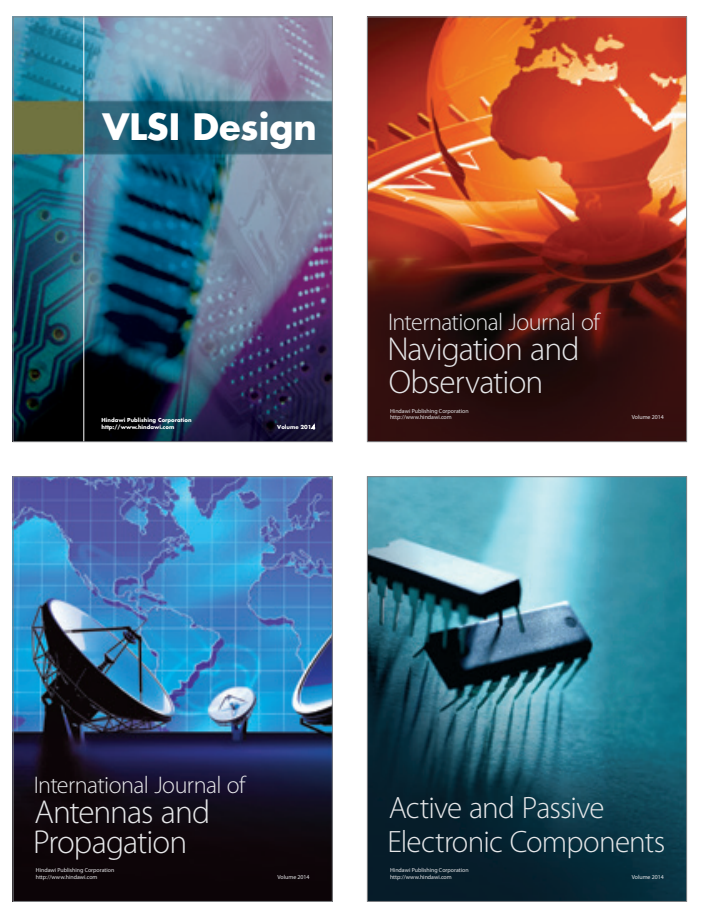
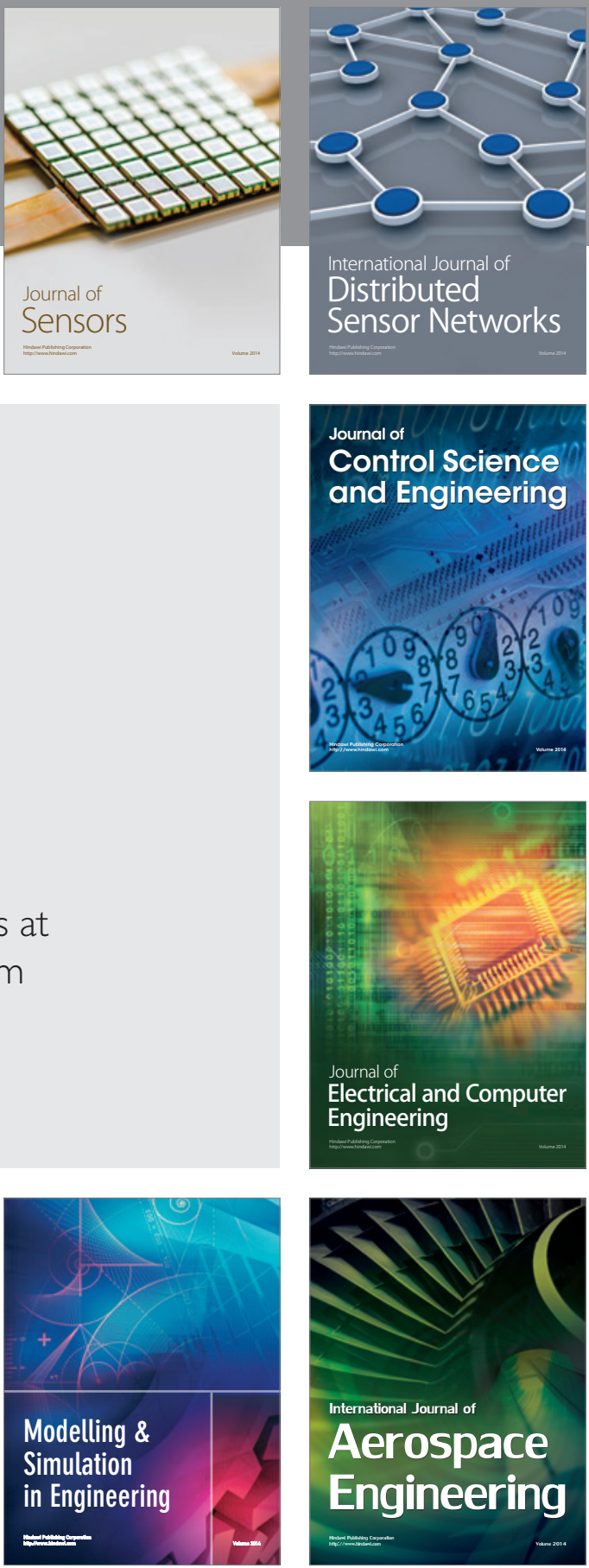

Journal of

Control Science

and Engineering
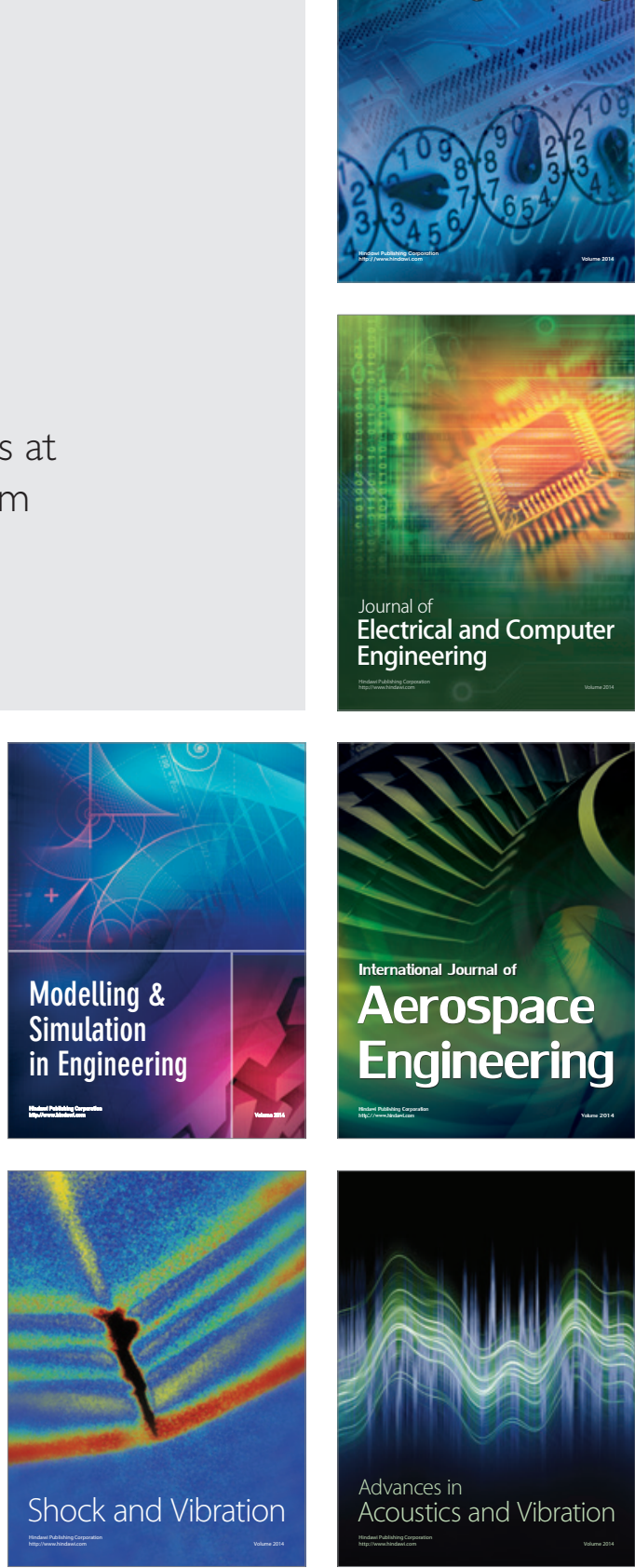